\title{
Induced rotation with concentric patterns
}

\author{
R. H. DAY \\ Monash University, Victoria 3168, Australia
}

\begin{abstract}
Duncker (1929) described induced rotation of a radial-line pattern when a concentric, enclosing annulus pattern rotated. This observation has not, so far, been confirmed or extended. Six experiments are described. The results from Experiments 1 and 2 showed that the frequency with which induced rotation is reported during standard observation periods is not affected by either angular velocity up to $15 \mathrm{deg} / \mathrm{sec}$ or unpatterned gaps up to $5 \mathrm{deg}$ wide between the inner and outer patterns. Experiment 3 confirmed that the strength of the effect can be satisfactorily measured by cancellation of induced movement. Experiments 4-6 showed that induced rotation is very weak or absent when the inner disk rotates and the concentric annulus is stationary, increases in velocity as the number of radial lines in the rotating annulus increases by up to half the number in the stationary disk, and is only slightly stronger when the area of contrast between moving and stationary lines is poorly resolved in the peripheral visual field. The results are considered in terms of the resolution in perception of displacement ambiguity between moving and stationary elements.
\end{abstract}

Duncker (1929) briefly described some observations of induced rotation of a stationary patterned disk by actual rotation of a concentric patterned annulus surrounding it. The inner disk and concentric annulus were made of gray card. The patterns consisted of regularly spaced black, radial lines. In the first part of Duncker's experiment, subjects simply reported their impressions of the inner pattern when the outer one rotated. The reports indicated that apparent rotation of the inner, stationary pattern was observed along with perceived rotation of the annulus. In the second part of the experiment, the strength of the induced effect was measured in terms of its apparent velocity, using the cancellation or "nulling" method; the inner pattern was rotated in the direction opposite to its apparent rotation to cancel it. Duncker reported that induced rotation was strongest when rotation of the annulus was less than $5 \mathrm{deg} / \mathrm{sec}$ viewed from $30 \mathrm{~cm}$. At $15 \mathrm{deg} / \mathrm{sec}$, the induced effect was virtually negligible. Induced rotation was also weakened when the "clarity of contact" between the inner and outer patterns was reduced by high angular velocities of the latter. High velocities resulted in fusion or near-fusion of the radial lines. A similar weakening of the effect occurred when the region of contact was located in the peripheral visual field.

As far as is known, there have been no investigations of induced rotation since Duncker's original study. In addition to the desirability of confirming and extending Duncker's rather perfunctory observations, two considerations would seem to justify fur-

The assistance of Jane Millar and Rod Dickinson in the collection and analysis of data is gratefully acknowledged. This program was supported by the Australian Research Grants Committee. ther exploration of the effect. First, in the original experiments only the outer annulus was rotated, while the inner disk was stationary. There was no attempt to establish whether induced rotation of the annulus occurred when the disk was rotated. Given Duncker's strong emphasis on the "principle of enclosure," the claim that an enclosed element is induced to move by real motion of the enclosing frame or field, this omission is surprising. Second, Duncker's experiments on induced rotation, as far as can be established from his brief report, were conducted in normal room illumination. In this condition, induced linear movement would be most unlikely to occur unless the moving field were very large. With small fields, stationary visible references would markedly reduce or eliminate the induced effect.

The six experiments described here were carried out in order to check Duncker's (1929) observations and to investigate the effects of separation between inner and outer patterns, enclosure and nonenclosure by the rotating pattern, the number of outer radial lines relative to the inner, and the location of the region of contact between the two patterns in the peripheral visual field. Since induced rotation has not so far been investigated in detail, these experiments were exploratory rather than 'systematic. Further detailed experiments are necessary to establish the functional relationships between various stimulus parameters and the strength of induced rotation.

\section{EXPERIMENT 1: FREQUENCY OF REPORT OF INDUCED ROTATION}

In his account of induced rotation, Duncker (1929) described induced rotation of a stationary, patterned 
disk by actual rotation of a concentric, rotating annulus without giving any details of the number of observers or the frequency with which the effect was reported. The purpose of the first experiment was to confirm the occurrence of the effect simultaneously with perceived rotation of the outer pattern and to establish the frequency of its occurrence over a range of inducing velocities. Because of the brevity and sparseness of Duncker's description, no attempt was made to repeat in detail the conditions under which his observations were made. It is reasonable to suppose that the effect is sufficiently robust to occur over a range of stimulus conditions.

\section{Method}

Subjects. There were 12 volunteer subjects, 6 men and 6 women, all of whom were undergraduates or graduate students who were paid for their services.

Apparatus. Two radially patterned concentric disks could be independently rotated clockwise $(\mathrm{CW})$ or counterclockwise (CCW) between 0 and $18 \mathrm{deg} / \mathrm{sec}$ by means of silenced synchronous motors operating independently through belt drives. Concentricity and independence of direction and angular velocity were achieved by passing the axle of the smaller, inner disk through the tubular axle of the larger disk. The smaller, inner pattern was $8 \mathrm{~mm}$ in front of the larger. Both axles passed through the center of a rectangular screen of gray cardboard, the same material from which the disks were made. This screen measured $101 \mathrm{~cm}$ wide $\times 81 \mathrm{~cm}$ high and formed a background to the concentric stimulus arrangement. The luminance of the background and the gray areas of the disks was $49 \mathrm{~cd} / \mathrm{m}^{2}$. A chinrest clamped to a table controlled the distance between eyes and rectangular screen at $57 \mathrm{~cm}$. The small disk was $.5 \mathrm{~cm}$ nearer and the large disk $1.3 \mathrm{~cm}$ nearer. At $57 \mathrm{~cm}$, $1 \mathrm{~cm}$ at the screen subtended a visual angle of $1 \mathrm{deg}$. Thus, $1 \mathrm{~cm}$ at the disks subtended slightly less than $1 \mathrm{deg}$. The beginning and end of an observation trial, that is, the period during which the subject viewed the concentric stimulus pattern before making a report, was signaled by two "pips" from a sound generator.

Stimulus patterns. The concentric patterns are shown in Figure $1 \mathrm{~A}$. The inner pattern was $4 \mathrm{~cm}$ in diameter, and the surrounding annulus $8 \mathrm{~cm}$ radially. These were the same as those in Duncker's experiment but viewed from a greater distance, $57 \mathrm{~cm}$ rather than $30 \mathrm{~cm}$. The patterns on both central disk and surrounding annulus consisted of 32 regularly spaced, radial lines $.5 \mathrm{~mm}$ thick. The inner pattern was stationary throughout. The larger pattern rotated in the $\mathrm{CW}$ direction at one of three angular velocities: $4,8.7$, or $15 \mathrm{deg} / \mathrm{sec}$. Although these were the same angular velocities used by Duncker (1929), the greater viewing distances in this experiment, $57 \mathrm{~cm}$ rather than $30 \mathrm{~cm}$, rendered them slower in linear terms. Nevertheless, their range extended beyond the inducing velocity $(5 \mathrm{deg} / \mathrm{sec})$ at which Duncker stated induced velocity was weakened.

Procedure. The subjects were required to describe the appearance of the concentric pattern during 3-sec observation periods.
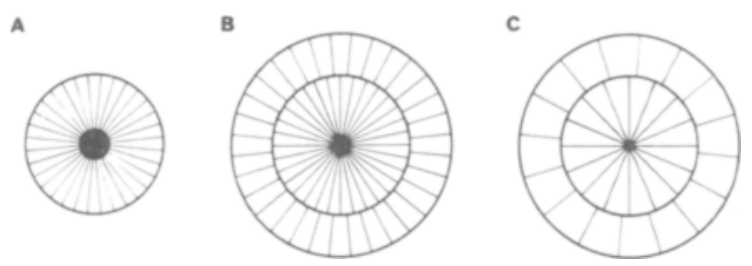

Figure 1. Examples of the concentric stimulus patterns for the six experiments. A was used in Experiment 1, and B in Experiment 3; $\mathbf{C}$ represents one of the stimulus arrangements for Experiment 5 .

Neither movement nor stationariness were mentioned in the instructions. If rotation was reported, the subjects were then asked to report the region of the concentric pattern in which it occurred and its direction. At the beginning of a session, each subject was directed by standard, recorded instructions to place his or her chin on the rest, close his or her eyes until the first pip was heard, then to open the eyes and observe and report the appearance of the pattern, and, finally, to close the eyes again. There were 15 such trials, 5 at each of the three angular velocities of the annulus. The order of the 15 trials was differently randomized for each subject.

\section{Results and Discussion}

All subjects reported $\mathrm{CW}$ rotation of the outer pattern. The total and mean frequencies of apparent rotation and stationariness (St) of the inner pattern are shown in Table 1. There was a total of 60 reports for each condition. The expected direction of induced rotation was CCW. It can be seen that this direction predominated at each velocity. The remainder of the reports were St. There were no reports of $\mathrm{CW}$ rotation.

An analysis of variance of the mean frequencies showed that the difference between the means was not significant $[\mathrm{F}(2,22)=.83, \mathrm{p}>.05]$.

The outcomes of this experiment show that both real movement of the outer patterned annulus and induced movement of the inner disk in the opposite direction occur together, as Duncker (1929) stated. Inspection of individual reports indicated that if subjects induced rotation in one of the three conditions, they tended to do so in the others. That is, there was a consistency of reporting across conditions. No subject failed completely to report induced movement. The frequency of the effect did not vary with the angular velocity of the annulus. At the highest velocity (15 deg/sec), there was no indication that induced rotation was markedly weakened, as indexed by lower

Table 1

Total and Mean Frequencies of Report of Counterclockwise Rotation (CCW), Stationariness (St), and Clockwise Rotation (C) of the Stationary Inner Pattern (Figure 1) for Three Angular Velocities of the Inner, Stationary Pattern in Experiment 1

Velocity (in Degrees per Second)

\begin{tabular}{|c|c|c|c|c|c|c|c|c|c|}
\hline \multirow[b]{2}{*}{ Frequency } & \multicolumn{3}{|c|}{4} & \multicolumn{3}{|c|}{5.73} & \multicolumn{3}{|c|}{15} \\
\hline & $\mathrm{CCW}$ & St & $\mathrm{CW}$ & $\mathrm{CCW}$ & St & $\mathrm{CW}$ & $\mathrm{CCW}$ & St & $\mathrm{CW}$ \\
\hline $\begin{array}{l}\text { Total } \\
\text { Mean }\end{array}$ & $\begin{array}{r}45.00 \\
3.75\end{array}$ & $\begin{array}{r}15.00 \\
1.25\end{array}$ & $\begin{array}{l}0 \\
0\end{array}$ & $\begin{array}{r}41.00 \\
3.41\end{array}$ & $\begin{array}{r}19.00 \\
1.58\end{array}$ & $\begin{array}{l}0 \\
0\end{array}$ & $\begin{array}{r}38.00 \\
3.17\end{array}$ & $\begin{array}{r}22.00 \\
1.83\end{array}$ & $\begin{array}{l}0 \\
0\end{array}$ \\
\hline
\end{tabular}

Note-The maximum total was 60 and the maximum mean was 5 . 
frequency of report, as could be expected from Duncker's (1929) report. Even though the velocity of movement in linear terms was less than in Duncker's experiment, it nevertheless extended well into the range in which he found induced rotation to be weak.

\section{EXPERIMENT 2: SEPARATION BETWEEN MOVING AND STATIONARY LINES}

In the experiments described by Duncker (1929), the moving and stationary radial lines were more or less continuous when in alignment. That is, the outer ends of the stationary lines and the inner ends of the moving lines fell on the same circle defined by the circumference of the stationary pattern. Thus, there was no gap radially between the two patterns. Is it necessary that the ends of the moving and stationary lines of the patterns be contiguous, or nearly so, for induced motion to occur? Alternatively, is induced motion weakened by frequency of report when a gap occurs between the inner and outer patterns? The second experiment was intended to answer these questions. There were three conditions: no gap, a gap of $1 \mathrm{~cm}$, and a gap of $5 \mathrm{~cm}$. The subjects were required to describe the appearance of the inner pattern and, if rotation was reported, to indicate its direction.

\section{Method}

Subjects. There were 12 new subjects, 4 men and 8 women.

Apparatus. The apparatus, viewing distance, and observation periods were the same as in the first experiment.

Stimulus patterns. The inner, stationary pattern was $4 \mathrm{~cm}$ in diameter and consisted of 32 equally spaced radial lines, as in the first experiment. The outer annuli were $8 \mathrm{~cm}$ radially and consisted also of 32 equally spaced lines. For one concentric pattern, there was no gap between the inner and outer lines (that is, the arrangement was the same as in Experiment 1), for another there was a 1-cm gap, and for a third there was a $5-\mathrm{cm}$ gap. The gaps in each case formed a gray annulus between the patterns. It should be noted that the wider the gap, the more peripheral the inducing pattern in the visual field; that is, gap size and location of the outer pattern were confounded. The outer pattern rotated at $6 \mathrm{deg} /$ sec.

Procedure. The procedure was essentially the same as in Experiment 1 , with each subject undergoing five observation trials of 3 sec duration for each of the three concentric patterns. The subjects were required to report the appearance of the inner pattern. If apparent rotation was reported, they were required to report its duration. The 15 trials were randomized differently for each subject. The expected direction of induced movement was CCW.

\section{Results and Discussion}

There was a total of 60 trials in each condition. The totals and mean frequencies of $\mathrm{CCW}, \mathrm{CW}$, and
St reports are shown in Table 2 . It can be seen that $\mathrm{CCW}$ reports were again the most frequent, followed by $\mathrm{St}$ reports. There were no reports of $\mathrm{CW}$ movement. An analysis of variance showed that the differences between the three means was not significant $[F(2,22)=2.24, p>.05]$. Thus, discontinuities between the inner and outer radial lines did not affect the frequency with which induced movement of the former was reported. While it is conceivable that the strength of induced rotation might vary in consequence of such gaps, the abutment of line ends in the two patterns is clearly not necessary for its occurrence.

\section{EXPERIMENT 3: QUANTITATIVE OBSERVATIONS}

The purpose of the third experiment was to establish the strength of induced rotation in terms of its apparent velocity, using counterrotation of the inner pattern to cancel it.

\section{Method}

Subjects. A group of 12 new subjects, 4 men and 8 women, drawn from the same groups as the earlier subjects, participated.

Apparatus. There were two additions to the apparatus described above. The first was an opaque, rectangular screen, $90 \mathrm{~cm}$ wide $\times 61 \mathrm{~cm}$ high, with a $1.6-\mathrm{cm}$ viewing aperture at its center. The purpose of the screen and aperture was to exclude other stationary objects in the room from view and to make it easier for the experimenter to adjust the starting velocity of the inner pattern out of the subject's view. The screen was clamped to a table in front of the subject so that the viewing distance from eye to screen was $57 \mathrm{~cm}$. The second addition was two rotary controls at the end of cables, by means of which the angular velocity of the inner and outer patterns could be varied between 0 and $18 \mathrm{deg} / \mathrm{sec}$. Angular velocities were continuously registered in separate digital displays visible only to the experimenter.

Stimulus patterns. The inner pattern was $20 \mathrm{~cm}$ in diameter, and the outer pattern $6 \mathrm{~cm}$ radially. These were the dimensions of the concentric arrangement in Duncker's (1929) cancellation experiment. However, his viewing distance was $30 \mathrm{~cm}$, so the visual angles would have been much greater. Each pattern again consisted of 32 equally spaced black radial lines. The outer pattern rotated at $8.7 \mathrm{deg} / \mathrm{sec}$. Viewed through the aperture, the concentric patterns were surrounded by a gray background of about the same radial width as the outer pattern. The circular contour of the aperture was blurred and indistinctly visible in the peripheral visual field.

Procedure. The subjects were required to cancel the induced rotation of the inner pattern by rotating it in the opposite direction. The duration of each trial was $6 \mathrm{sec}$. Preliminary tests had shown that this was the minimum period in which the task could be carried out with confidence. There were 10 trials, 5 in which the velocity of the inner disk had to be decreased from $12 \mathrm{deg} / \mathrm{sec}$ (descending trials) and 5 in which it had to be increased from stationariness

Table 2

Total and Mean Frequencies of Report of Counterclockwise (CCW), Stationariness (St), and Clockwise Rotation (CW) of the Stationary Inner Pattern for Three Radial Extents of Unpatterned Gap Between the Moving and Stationary Patterns

Gap (in Centimeters)

\begin{tabular}{crrrrrrrrrr} 
Frequency & $\mathrm{CCW}$ & $\mathrm{St}$ & $\mathrm{CW}$ & $\mathrm{CCW}$ & $\mathrm{St}$ & $\mathrm{CW}$ & $\mathrm{CCW}$ & $\mathrm{St}$ & $\mathrm{CW}$ \\
\hline Total & 46.00 & 14.00 & 0 & 51.00 & 9.00 & 0 & 42.00 & 18.00 & 0 \\
Mean & 3.83 & 1.17 & 0 & 4.25 & .75 & 0 & 3.50 & 1.50 & 0 \\
\hline
\end{tabular}


(ascending trials). The order of the 10 trials was differently randomized for each subject. Before the first trial, the subjects were instructed to adjust the inner pattern until it appeared to be stationary. The subjects were given 2 practice trials before the 10 experimental trials. The point of fixation was not rigorously controlled. The subjects were told simply to look at the inner pattern.

\section{Results and Discussion}

The subjects experienced little or no difficulty in cancelling induced rotation by actual rotation of the inner pattern in the opposite direction. All cancellations were $\mathrm{CW}$ in direction. The mean angular velocity necessary for cancellation was $2.41 \mathrm{deg} / \mathrm{sec}$ (SD: .19). It can be noted that, for the same angular velocity, the mean in Duncker's (1929) experiment was $5.6 \mathrm{deg} / \mathrm{sec}$. However, the linear velocity would have been greater since the viewing distance was less. The results show that cancellation of induced rotation by counterrotation could be satisfactorily and confidently carried out by the observers.

\section{EXPERIMENT 4: THE EFFECTS OF ENCLOSURE AND NONENCLOSURE}

One of Duncker's (1929) general conclusions regarding induced movement was that the enclosure of a stationary element by a moving frame or field is a primary determinant of the effect. He argued that movement of the field when its velocity is below the subject-relative movement threshold is imputed to the smaller, stationary element. More recently, this generalization, based on observations of induced linear movement, has been called into question. Either induced movement of the stationary, enclosed element or real movement of the surrounding field is perceived (Day, 1978; Day, Millar, \& Dickinson, 1979; Rock, Auster, Schiffman, \& Wheeler, 1980).

Duncker did not test his "principle of enclosure" with concentric patterns, although this arrangement would seem ideally suited to doing so. The purpose of the fourth experiment was to establish whether induced rotation of the outer, stationary pattern occurs when the inner pattern rotates and, if so, whether the effect is as strong as for that of the inner pattern when the outer rotates. Informal preliminary observations indicated that induced rotation of the outer pattern was either hardly discernible or completely absent. It was thought that this might be due to the smaller area of the inner pattern relative to the other. For this reason, the inner disk was made greater in area than the annulus.

\footnotetext{
Method

Subjects. There were again 12 subjects, 7 men and 5 women, drawn from the same sources as before. None had taken part in earlier experiments.

Apparatus. The apparatus was the same as for Experiment 3.

Stimulus patterns. The inner pattern was $20 \mathrm{~cm}$ in diameter, and the annulus pattern was $2.8 \mathrm{~cm}$ wide radially. The area of the inner disk was therefore $314 \mathrm{~cm}^{2}$, and of the annulus, $200 \mathrm{~cm}^{2}$. The ratio between the areas of the inner and outer patterns was $1.57: 1$, the
}

reverse of that in Experiment 3, in which the annulus was greater in area.

Procedure. Each subject cancelled induced rotation of the inner pattern when the outer pattern rotated at $6 \mathrm{deg} / \mathrm{sec}$ (condition I) and induced rotation of the outer pattern when the inner pattern rotated at $6 \mathrm{deg} / \mathrm{sec}$ (condition $\mathrm{O}$ ). For half the subjects, the order was IO, and for the other half, OI. There were 10 trials of $6 \mathrm{sec}$ duration in each condition, 5 ascending trials and 5 descending trials from the same starting points as those in Experiment 3. The order of the 10 trials was again randomized separately for each observer. Instructions and practice were essentially similar to those for Experiment 3. The point of fixation was not rigorously controlled. The subjects were simply asked to look at either the inner or the outer pattern, as appropriate.

\section{Results and Discussion}

The mean angular velocity for cancellation of induced rotation for condition I was $2.16 \mathrm{deg} / \mathrm{sec}$ (SD: .72 ), and for condition $O, .36 \mathrm{deg} / \mathrm{sec}$ (SD: .24). The range of individual means for condition I was .78-2.70 $\mathrm{deg} / \mathrm{sec}$, and for condition $\mathrm{O}, .12-.66 \mathrm{deg} / \mathrm{sec}$. The difference between the means was significant $[\mathrm{t}(11)=$ $9.74, \mathrm{p}<.005]$.

While there is no doubt that induced rotation of the inner pattern was markedly stronger than that of the outer pattern, the question arises as to whether induced movement of the annulus occurred at all, that is, whether the slight effect was artifactual. The rotary control operated by the subject allowed for movement of the pattern only in the direction opposite to its induced movement. The pattern could not be moved in the same direction. Given that subjects were instructed to manipulate the control to cancel induced movement, and, furthermore, given that any movement, no matter how slight, must have occurred in the direction of cancelling induced rotation, it is conceivable that the effect for condition O may simply have been an artifact of instructions and the unidirectionality of cancelling movement. Whether or not this was so remains to be shown. Either way, induced rotation of the outer concentric pattern by rotation of the inner pattern was very weak indeed compared with that of induced rotation of the inner pattern by rotation of the outer.

\section{EXPERIMENT 5: EFFECT OF RELATIVE NUMBER OF INDUCING LINES}

In the experiments described so far, the inner and outer patterns consisted of the same number of radial lines. It is conceivable that the number of lines in the inducing pattern relative to that in the stationary pattern determines in part the apparent velocity of induced rotation. That is, the velocity of induced rotation may be attributable in part to the frequency with which the outer moving lines pass or "shear" relative to the stationary inner lines. This possibility was investigated, in the fifth experiment, with outer inducing patterns consisting of $2,4,8$, and 16 radial lines and with an inner pattern consisting always of 16 lines. 
Method

Subjects. There were 12 subjects, 7 men and 5 women, 4 of whom had participated in one of the earlier experiments.

Apparatus. The apparatus was the same as in Experiment 4.

Stimulus patterns. One of the four patterns, that in which there were 16 lines in both patterns, is shown in Figure 1c. The inner pattern was $20 \mathrm{~cm}$ in diameter in all cases and consisted of 16 radial lines. The four outer patterns consisted of $2,4,8$, and 16 radial lines. The angular velocity of the outer pattern was $6 \mathrm{deg} / \mathrm{sec}$.

Procedure. Each subject cancelled induced rotation of the inner pattern under all four conditions. The order was different for each subject but balanced across the group. There were 5 ascending trials from stationariness and 5 descending from $12 \mathrm{deg} / \mathrm{sec}$ in each condition. These 10 trials were differently randomized for each subject.

\section{Results and Discussion}

The mean velocities for cancellation of induced rotation under the four conditions are shown in Figure 2. It can be seen that the apparent velocity of induced rotation increased relatively steeply as the number of outer radial lines increased from 2 to 8 and leveled off between 8 and 16 lines. It can also be seen that, while apparent velocity was lowest with 2 lines, induced rotation nevertheless occurred with a velocity of $1.23 \mathrm{deg} / \mathrm{sec}$.

A one-way analysis of variance showed that the number of lines in the outer pattern exercised a significant effect on the apparent velocity of induced movement $[F(3,33)=20.18, p<.01]$. Further analysis by means of the Newman-Keuls test showed that the differences between the velocities with 2 and 4 lines and those velocities with between 8 and 16 lines were not significant. However, the differences between the velocities with 2 and 16 lines and those between the velocities with 4 and 16 lines were significant, with $\mathrm{p}<.01$ in each case.

It is conceivable that the increase in apparent velocity with increase in the number of inducing lines is due to the "shearing" frequency of inner and outer lines. That is, the frequency with which outer lines shear in respect of inner lines determines apparent velocity up to a limiting frequency. It is conceivable that the upper limit is reached when differences in frequency are no longer discriminable. Thus, it could

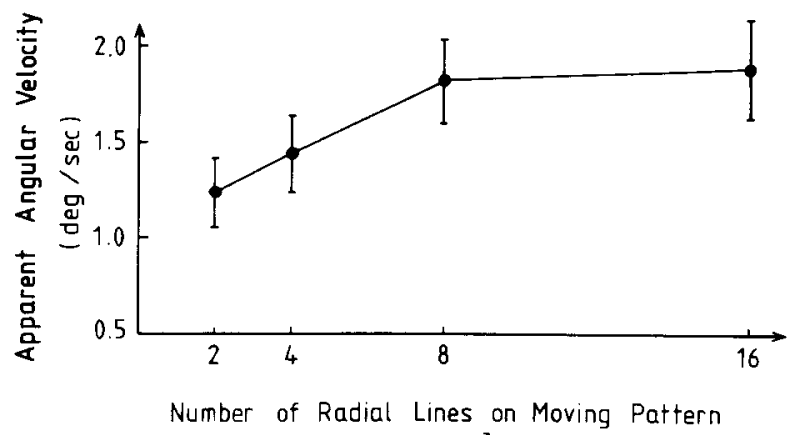

Figure 2. Mean angular velocities and standard deviations for cancellation of induced rotation of an inner 16-line pattern with outer patterns consisting of 2, 4, 8, and 16 radial lines in Experiment 5 . The stimulus patterns are shown in Figure 1. be expected that there would be a tradeoff between the frequency of elements in the outer pattern and the angular velocity.

\section{EXPERIMENT 6: EFFECT OF LOCUS OF FIXATION}

In his account of induced rotation, Duncker (1929) contended that if the "clarity of contact" between the two patterns is in any way reduced, induced rotation is thereby "jeopardized." He named two conditions which reduce clarity: high rotation velocities leading to fusion of the inducing elements and location of the circular contour between the two patterns in the peripheral visual field. Duncker's contention was examined in the sixth experiment. The inner pattern was fixated at either its center or at its circumference. In the first condition, the region of contact between the two patterns was located 6 deg eccentrically so that it was visible but poorly resolved, while in the second it was clearly resolved. It was expected that the apparent velocity of induced movement with central fixation of the inner pattern would be much less than that with eccentric fixation.

\section{Method}

Subjects. There were 12 subjects, 7 men and 5 women, drawn from the same sources as before; 6 of them had taken part in one earlier experiment.

Apparatus. The apparatus was the same as in Experiments 3, 4, and 5 , with the exception that the screen and viewing aperture were moved so that distance between background and aperture was $95 \mathrm{~cm}$. At this distance, $1 \mathrm{~cm}$ at the background subtended a visual angle of .6 deg. The increase in distance was necessary to locate the contour between the inner and outer patterns 6 deg eccentrically when the center of the pattern was fixated. At this angle, the pattern in the region of contact was visible but hardly resolvable.

Stimulus patterns. The inner pattern was $20 \mathrm{~cm}$ in diameter, and the outer annulus was $6 \mathrm{~cm}$ radially, as shown in Figure 1B. Each pattern consisted of 32 equally spaced radial lines. The angular velocity of rotation was $6 \mathrm{deg} / \mathrm{sec}$.

Procedure. Each subject cancelled induced rotation while fixating the inner pattern at its center $(C)$ and at its circumference $(P)$. For half the subjects, the order was CP, and for the other half, PC. There were 5 ascending and descending trials for each condition from the same starting velocities of the inner pattern as before. The 10 trials were differently randomized for each observer.

\section{Results and Discussion}

The mean cancellation velocity of the inner pattern for condition C was $2.27 \mathrm{deg} / \mathrm{sec}$ (SD: .59), and for condition P, $2.56 \mathrm{deg} / \mathrm{sec}$ (SD: .62). The difference between these means proved to be significant $[t(11)=$ $2.39, \mathrm{p}<.05$ ]. Thus, induced rotation was slightly reduced by lack of clarity of the region of contact between the two patterns consequent on poorer visual resolution. Nevertheless, the reduction was small, and induced movement unquestionably occurred when the region of contact was located 6 deg eccentrically. It is, of course, conceivable that greater eccentricity would result in greater weakening of the induced effect. 


\section{GENERAL DISCUSSION}

The experiments confirm Duncker's (1929) early observation thát a stationary radially patterned disk appears to rotate in the direction opposite to a rotating, patterned annulus surrounding it. The first experiment confirmed that induced rotation occurs simultaneously with perceived real rotation of the outer annulus pattern. The results also show that the frequency with which induced rotation is reported does not vary with inducing velocities between 4 and $15 \mathrm{deg} / \mathrm{sec}$ viewed from $57 \mathrm{~cm}$ and when gaps of up to $5 \mathrm{deg}$ in visual angle occur between the inner and outer radial lines. The second of these two findings does not, of course, rule out the possibility that the effect is reduced in strength when it is indexed by apparent velocity. It merely shows that actual abutment of inner and outer lines is not a necessary condition for induced rotation to occur.

Experiment 3 confirmed that induced rotation can be measured by cancelling it with counterrotation. Using this method, it was shown that the effect is markedly stronger when the stationary pattern is enclosed by the annulus, the strength of the effect varies according to the number of radial elements in the outer annulus relative to that in the inner pattern up to a limiting ratio, and the strength of the effect is less, but not markedly so, when the contour between the two patterns is located 6 deg eccentrically in the visual field.

Two points can be made about these outcomes. First, while induced movement of the outer pattern, as indexed by counterrotation, occurred for all subjects when the inner pattern rotated, the effect was nevertheless very weak. Additional experiments are necessary to confirm whether, in fact, induced rotation of the outer pattern does occur. It is conceivable, of course, that the visible texture of the stationary background surrounding the annulus might serve as a reference and thereby weaken or eliminate altogether induced movement of the annulus when the inner pattern rotates. Second, induced rotation was weakened only slightly when the contour between the two circular patterns was located 6 deg eccentrically compared with when it was located centrally in the visual field. It remains to be shown whether greater weakening of induced movement occurs when the area of contact between the two patterns is more eccentric than $6 \mathrm{deg}$. However, it is to be noted that, at $6 \mathrm{deg}$, visual resolution of the radial line patterns is very much poorer than when the area of contact between the two patterns is foveal. To this extent, it cannot be said that induced rotation is markedly affected or "jeopardized" when the "clarity of contact" between the two patterns is reduced, as claimed by Duncker (1929).
It is useful to consider induced rotation in terms of the perceptual ambiguity of relative displacement between moving and stationary elements and the ways in which this ambiguity is resolved in perception.

Displacement is relative and, as such, is by itself ambiguous. When the velocity of movement is below the subject-relative threshold (Shaffer \& Wallach, 1966), and in the absence of external references, the displacement of one element relative to another can derive from movement of the first in one direction while the second is stationary, movement of the second in the opposite direction while the first is stationary, or movement of both in opposite directions. In the case of linear displacement between two points in a featureless field, the ambiguity is resolved by either of the points appearing to move while the other appears to be stationary (Day, 1978; Mack, Fisher, \& Fendrich, 1975). With a stationary point enclosed by a moving frame, the most widely investigated arrangement, either the point or the frame appears to move while the other element appears to be stationary (Day, 1978; Day, Millar, \& Dickinson, 1979) or, alternatively, both point and frame appear to move at the same time in opposite directions (Rock, Auster, Schiffman, \& Wheeler, 1980; Wallach, Bacon, \& Schulman, 1978).

These various forms of perceptual resolution have been shown to occur when the velocity of the moving element is below the subject-relative threshold. Of course, when velocity exceeds that threshold, relative displacement is no longer strictly ambiguous, since discriminable displacement relative to the observer himself occurs. Nevertheless, increases in velocity to values above the subject-relative threshold do not seem to affect the way in which displacement between the two display elements is perceived. In point of fact, Wallach, Bacon, and Schulman (1978) have recently noted that perceiving the motion of the surround does not necessarily diminish induced motion. It is as if there is a separation (Duncker, 1929; Wallach, 1959) between the object-relative and subject-relative motion systems. It seems reasonable, therefore, to consider the outcomes of the experiments reported here with rotating patterns in terms of the resolution of ambiguity, even though the angular velocity of the rotating element was above the subject-relative threshold.

With circular concentric patterns, perceptual resolution seems to involve only perceived rotation of both inner disk and outer annulus in opposite directions. When the annulus rotates, it is perceived as doing so and, at the same time, the inner pattern is seen to move in the opposite direction with a perceived velocity of between a quarter and a third of that of the annulus. When the inner disk rotates, it is also perceived as doing so, whereas the annulus appears either 
to remain stationary or to rotate only very slowly in the opposite direction. As pointed out above, this latter observation is in doubt and requires confirmation, preferably using methods different from those reported here.

Thus, the perceptual resolution of displacement between two concentric patterns involves perceived real movement of the rotating element and induced rotation of the stationary element in the opposite direction. Unlike the point and frame display, there is no evidence that when the surrounding element, the equivalent of the frame, moves it is sometimes perceived to be stationary, with perceived movement confined entirely to the disk. Nevertheless, the observation that induced movement of the annulus is, at the most, very weak while that of the disk is consistently strong firmly supports Duncker's (1929) conclusion that enclosure of one element by another is among the salient determinants of induced movement.

Two points can be made in conclusion. First, throughout the series of experiments described here, induced rotation was measured using the cancellation method. Although there is no reason to doubt the reliability of this method, confirmation of the outcomes using an alternative technique, such as matching, is clearly desirable. Second, it is emphasized again that these experiments were exploratory and probing rather than systematic in regard to a particular variable. Further work is essential to establish the main determinants of the effect and its functional relationships.

\section{REFERENCES}

DAY, R. H. Induced visual movement as nonveridical resolution of displacement ambiguity. Perception \& Psychophysics, 1978, 23, 205-209.

Day, R. H., Millar, J., \& Dickinson, R. G. Induced movement as nonveridical resolution of displacement ambiguity: Effect of enclosure and the number of field elements. Perception \& Psychophysics, 1979, 25, 23-28.

Duncker, K. Induzierte Bewegung (Ein Beitrag zur Theorie optisch Wahrgenommener Bewegung). Psychologische Forschung, 1929, 12, 180-259. (Translated as "Induced motion," in W. D. Ellis (Ed.), A source book of Gestalt psychology. London: Paul, Trench \& Trubner, 1938.)

Mack, A., Fisher, C. G., \& Fendrich, R. A re-examination of two-point induced movement. Perception \& Psychophysics, 1975, 17, 273-276.

Rock, I., Auster, M., Schiffman, M., \& Wheeler, D. Induced movement based on subtraction of motion from the inducing object. Journal of Experimental Psychology: Human Perception and Performance, 1980, 6, 391-403.

ShafFer, O., \& Wallach, H. Extent of motion thresholds under subject-relative and object-relative conditions. Perception \& Psychophysics, 1966, 1, 447-451.

Wallach, H. The perception of motion. Scientific American, $1959,201,56-60$.

Wallach, H., Bacon, J., \& Schulman, P. Adaptation in motion perception: Alteration of induced motion. Perception \& Psychophysics, 1978, 24, 509-514.

(Received for publication November 7, 1980; revision accepted March 23, 1981.) 